\title{
DNA Replication of Single Cell Using Laser Heating in Varied Osmotic Solutions
}

Min-Sheng Hung and Ying-Ting Shih

Department of Biomechatronic Engineering, National Chiayi University, Chiayi, Chiayi, Taiwan (Republic of China)

DNA encodes the genetic information of all the inheritable characteristics of an organic structure. In a previous study, we proposed and demonstrated a microfluidic platform integrated with laser-induced heating for real-time replication and detection of IDNA [1, 2]. Avidin-biotin binding was utilized to immobilize one end of the replicated products to the microchannels for the target sequences detection following replication. Our method enables rapid heating and cooling and thus can be applied to microanalysis systems for analyzing microliter samples such as those at the single-cell level. Single-cell analysis technology for rapid and low-cost target gene detection is a current prominent field of biomedical research. Accurately and rapidly replicating and detecting DNA sequences from a single cell is a key technical challenge in single-cell amplification and library preparation [3]. In the present study, we developed a platform with integrated laser heating for in situ target DNA replication and detection in a single cell. The following method was developed for single cell lysis and the target DNA replication. First, an infrared (IR) laser with a wavelength of $1.4 \mathrm{~mm}$ was applied to irradiate the cell for DNA release. Then, single cell lysis was conducted in various osmotic solutions to enable DNA release. Finally, the laser was used as a heating source for cellular DNA replication. Figure 1(a) and 1(b) displays the cells after disruption with a $0.4-\mathrm{W}$ laser in a hypertonic and hypotonic solution, respectively. The cell size and DNA fluorescent range (not shown) decreased after disruption in the hypertonic solution (Fig. 1(a)). By contrast, the cell burst and its substrate was released following disruption in the hypotonic solution (Fig. 1(b)). This result enabled the replication and detection of target DNA in a single cell. In addition, the relationship between cell disruption and laser power in various osmotic solutions is discussed. The techniques developed in this study can be applied to targeted gene detection at the single-cell level.

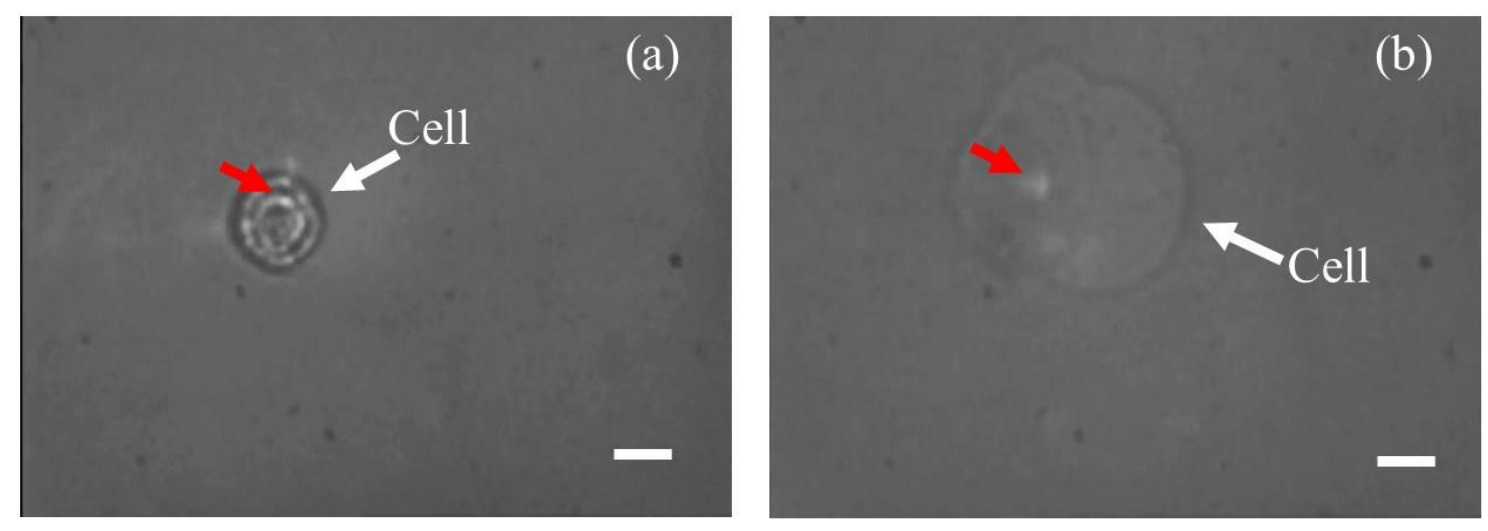

Figure 1. The image of cell disrupt at a $0.4-\mathrm{W}$ laser in the hypertonic solution (a) and in the hypotonic solution (b). (The red arrow indicates the position of laser spot. Scale bar 10 micrometer.)

References

[1] M.-S. Hung, C.-C. Ho, C.-P. Chen, Laser-induced heating integrated with a microfluidic platform for real-time DNA replication and detection, J. Biomed. Opt. 21 (2016) 087003.

[2] M.-S. Hung, C.-P. Chen, Laser-induced heating for in situ DNA replication and detection in microchannels, IET Nanobiotechnol. 12 (2018) 841-845. 
[3] I.C. Macaulay, C.P. Ponting, T. Voet, Single-cell multiomics: multiple measurements from single cells, Trends Genet. 33 (2017) 155-168. 